\title{
Des régularités sémantico-syntaxiques sous-jacentes
}

\author{
Decobert Bernard \\ Université de Limoges \\ CeReS - Centre de Recherches Sémiotiques \\ bernard.decobert@etu.unilim.fr \\ bernard.decobert@wanadoo.fr
}

\begin{abstract}
Ces travaux font l'objet d'une thèse qui sera consultable en 2015. Ils portent sur la syntaxe fondamentale et la prédictibilité syntagmatique. En ce sens, ils s'attachent à décrire et comprendre un phénomène qui croise plusieurs disciplines, la linguistique en général et la sémiotique bien sûr, mais audelà, et notamment, la lexicologie ou metalexicologie constitutive de lexiques électroniques pour le traitement automatique de la langue, la psycholinguistique et, manifestement aussi, le domaine très spécialisé des neurosciences cognitives. Dans une première partie nous décrivons les principales caractéristiques d'un modèle metaconceptuel tel qu'il nous apparaît. Dans une seconde nous entrons dans le détail des textes et montrons l'étendue du champ d'expression susceptible de justifier l'existence de récurrences metaconceptuelles.
\end{abstract}

\section{L'approche metaconceptuelle, aperçu général}

Bernard Victorri (1994) rappelait que «toute tentative de formalisation de la sémantique linguistique se trouve confrontée à deux problèmes essentiels. D'abord un problème de représentation : comment représenter le sens des unités linguistiques significatives (les monèmes d'André Martinet) et audelà le sens des expressions linguistiques issues de la combinaison de ces unités : syntagmes, énoncés ? Ensuite, un problème de calcul de ces représentations (...)». Il opposera ainsi deux méthodes, celle des grammaires formelles classiques qui revient à ramener ce calcul « de manière plus ou moins directe aux règles de composition algébrique entre des entités logiques (prédicats, opérateurs, formules) » et celle des modèles morphodynamiques, inspirés des travaux de René Thom, qui refusent la compositionnalité classique pour s'appuyer davantage sur une interaction dynamique complexe entre tout et parties, souvent inspirée d'ailleurs de la théorie gestaltiste (Victorri, 1994).

C'est en ouvrant plus encore le champ de la philosophie que Jean Petitot ${ }^{1}$ introduit une vision morphodynamique. Il postule qu'il existe « des infrastructures topologico-dynamiques sous-jacentes aux organisations tant syntagmatiques que paradigmatiques»; Et, déclare insuffisante «la description formaliste (logico-combinatoire et algébrique) des structures et de leurs fonctions ». Il faut lui adjoindre, dit-il, une explication morphodynamique des processus de formation de ces structures, "processus que l'on doit considérer comme des phénomènes naturels ».

Bien que se situant dans une orientation clairement morphodynamique, le travail présenté s'écarte de l'approche petitotienne en ce sens qu'il ne s'appuie pas sur un modèle mathématique pour tenter une formalisation de la sémantique linguistique ${ }^{2}$. Au contraire, on peut même considérer qu'il inverse cette proposition car c'est à partir de régularités observées empiriquement dans l'articulation de certaines catégories sémantiques qu'il propose un modèle mathématisable.

Dans notre thèse, nous donnons les caractéristiques générales d'un modèle conceptuel ou «metaconceptuel ». Nous décrivons aussi les étapes expérimentales statistiques et comparatives qui ont amené à faire l'hypothèse que le langage tend naturellement à se structurer autour et/ou à partir d'une chaîne sous-jacente, non aléatoire, hiérarchique, contraignante et restreinte de catégories sémantiques fondamentales. En d'autres termes, nous faisons l'hypothèse que le discours subit l'influence de 
constructions récurrentes sémantico-syntaxiques sous-jacentes qui en oriente le sens; tout notre travail revenant à montrer qu'un tel paradigme peut légitimement être postulé.

La notion de catégorie sémantique (CS) rejoint pour partie celle des concepts primitifs (semantics primes) d'Anna Wierzbicka (1996) en Natural Semantic Metalanguage (NSM). Les catégories sémantique - ou Métaconcepts (voire simplement concepts parfois) dans notre terminologie - tout comme les concepts primitifs en NSM sont réductibles à des mots clés. Toutefois, malgré des ressemblances évidentes, nos catégories sémantiques se différencient des concepts primitifs d'Anna Wierzbicka en particulier parce qu'elles tendent à s'articuler entre elles comme des étapes d'un seul et même processus. Nous retrouvons ici un modèle linéaire comparable avec celui des fonctions narratives chez Vladimir Propp (1970).

L'étude s'est attachée à repérer et modéliser ces catégories sémantiques qui relèvent a priori d'un système organisé. Elle cherche à en cerner la nature, la portée et les mécanismes inductifs et/ou logiques susceptibles de les incrémenter. Elle pose la question de la quantification du caractère contraint d'une telle structure conceptuelle hiérarchisée par rapport au hasard, aborde les prémices d'une grammaire permettant de décrire formellement ces contraintes, s'interroge sur le bien-fondé et la précision définitionnelle du catalogue de CS retenu, et engage une réflexion sur un traitement algorithmique des données observées.

\subsection{L'exclusion du choix conscient des auteurs}

Le Modèle Conceptuel part du constat, réalisé à partir de plusieurs milliers d'énoncés en français, d'abord de toutes provenances confondues, puis plus particulièrement sur des articles de presse, que des mêmes catégories sémantiques fondamentales tendent à s'ordonner selon une séquence déterminée. A l'analyse des résultats, l'étude conclut que de telles séquences ordonnées ne participeraient pas d'un choix délibéré des auteurs mais résulteraient vraisemblablement de schémas mentaux profondément ancrés ${ }^{3}$. Quatre constats concourent en effet à interpréter la présence d'un alignement répétitif de catégories sémantiques sous la forme d'une séquence type comme excluant le choix conscient des auteurs :

a. Les catégories sémantiques qui forment séquences dans les textes, sont révélées autant par la présence d'occurrences spécifiques que par celle d'occurrences impromptues (homonymes, homophones, symboles...).

b. Il n'apparaît pas, en général, de sélection objective, c'est-à-dire déterminante au sens hjelmslevien (Hjelmslev, 1996), entre deux occurrences consécutives, relevant d'une même séquence.

c. Projetées sur un espace cartésien sous la forme d'un semis de points, les séquences décrivent des alignements rectilignes ou quasi-rectilignes. De plus, on remarque une régularité des intervalles entre les principales occurrences constitutives de séquences (Figure 2).

d. Enfin, les séquences transparaissent indépendamment des césures phrastiques.

\subsection{Les catégories dans le modèle conceptuel}

On définit ordinairement une catégorie comme un ensemble d'éléments possédant en commun un trait distinctif. Le trait distinctif dans notre modèle est de nature géométrique et sémique. Il faut ajouter que, tout comme Louis Hjelmslev, nous définissons la catégorie comme un paradigme dont les éléments ne peuvent être introduits que dans certaines positions de la chaîne syntagmatique, à l'exclusion d'autres (Greimas \& Courtès, 1979 : 34).

\subsubsection{Concernant le trait distinctif}

Certains mots tendent à se regrouper. C'est le cas par exemple des collocations en linguistique. Lorsque la collocation n'autorise aucune variation de ses éléments, on parle aussi d'expression ou de locution figée 
comme : passer son tour ou l'union fait la force. Si maintenant on décide de décomposer ces deux expressions par exemple, en unités distinctes c'est-à-dire de les considérer hors contexte puis de les réduire à des «approximations schématiques » c'est-à-dire de tenter de rechercher les traits sémiques communs les plus simples et les plus généraux en les ramenant finalement à des concepts primaires ou à des géométries simples, il est toujours possible d'obtenir au moins trois grands visuels qui sont :

1. pour 'passer' $\rightarrow$ //l'idée d'un élément en mouvement, d'une trajectoire// $\equiv / / T R A J E C T O I R E / /$

2. pour 'tour' et 'union' $\rightarrow$ //l'idée de cercle ou d'éléments assemblés// $\equiv / / \mathrm{UNION} / /$;

3. et pour 'force' $\rightarrow / /$ l'idée d'un élément chargé ; chargé d'une puissance, d'un poids, d'un surcroît de quelque chose// $\equiv / /$ FORCE//.

Le procédé de « réduction schématique », en recherchant le concept type associable à telle ou telle lexie, et le décrivant comme un (ou plusieurs) élément pourvu de propriétés définitoires, se rapproche à la fois de l'analyse topologique et de la psychologie de la forme. On frôle également le schématisme kantien et l'on touche à l'analyse cognitiviste voire aussi au noème translinguistique de Bernard Pottier (1992).

Notre méthode oblige bien sûr à un sens élevé de l'abstraction mais c'est précisément notre démarche. La réduction schématique, dans le modèle conceptuel, constitue ainsi le trait distinctif de notre classe paradigmatique. Dans ce sens, elle se différencie des classes grammaticales telles que les définissent Algirdas Julien Greimas et Joseph Courtès (1979 : 33) : classes morphologiques (substantif, adjectif, verbe, etc.), syntaxiques (sujet, objet, prédicat, épithète, etc.), ou syntagmatiques (nominal, verbal). Cette distinction est fondamentale dans notre démarche. Dans le modèle conceptuel, seuls les traits sémiques en tant que concepts primaires sont recherchés. Ce qui élimine au moins dans un premier temps toute nécessité de trouver une justification conceptuelle aux classes grammaticales même universelles (nom, verbe, sujet, objet) comme dans la grammaire cognitive de Ronald Langacker ${ }^{4}$ par exemple. Au niveau de l'analyse initiale, sujet et prédicat n'ont plus aucune raison de s'opposer. Les concepts, en tant qu'unités sémiques, se différentient donc aussi du formalisme de la grammaire de Port Royal comme de celui du modèle standard de la grammaire générative de Noam Chomsky (1971).

\subsubsection{Récurrence et enchaînements linéaires}

A l'origine, notre approche empirique et intuitive nous a amené à considérer qu'il devait exister des récurrences schématiques et que tels lemmes se rapportaient davantage à telle unité de sens plutôt qu'à telle autre.

Si on prend par exemple les mots tour et union dans nos deux expressions précédentes et si on les considère sous l'angle de leur définition dans le dictionnaire général, tour et union se rapportent moins aux sèmes /trajectoire/ ou /force/ qu'à celui de /cercle/. Par ailleurs, si pour tour, le sème /cercle/ semble évident, il faut aussi considérer que pour union, il l'est aussi. Le dictionnaire général définira union par exemple comme un ensemble de différentes choses formant un seul tout. Par extension, on parlera de cercle d'amis, de jeu, ou encore de cercle familial. On voit dès lors qu'il est possible de ramener des groupes entiers de lexies à un même tronc commun conceptuel ${ }^{5}$.

De plus, et à partir de ce dernier exemple, il devenait possible d'aller plus loin et de supposer l'existence de continuités entre plusieurs expressions. Continuités qui s'opéreraient grâce à un élément commun, transitif, comme ici le sème /cercle/. Il s'agissait donc de chercher à observer si, dans les textes et discours, ce type de transitivité pouvait apparaître comme un principe général ou au contraire s'il n'était que fortuit; $\mathrm{Si}$, en définitive, le passage dans la chaîne syntagmatique entre le sème /trajectoire/ et le sème /force/, par exemple, impliquait nécessairement en son milieu le sème /union/ ou bien si ce dernier restait seulement occasionnel. Autrement dit, s'il existait des schèmes qui se repéraient sinon de manière systématique, au moins de manière récurrente comme nos trois concepts ci-dessus alignés dans cet ordre : trajectoire $\rightarrow$ cercle ou union $\rightarrow$ force. 
Cette question, très difficile, nécessitait que l'on élargisse la base lexicale. Il restait donc à extraire les bases lexicales nécessaires c'est-à-dire, repérer dans le lexique des unités susceptibles de contenir tel sème particulier, et dans un deuxième temps, grâce à l'analyse distributionnelle, d'étudier les types d'articulations possibles entre catégories.

\subsubsection{Les résultats}

A notre plus grand étonnement, non seulement il fut possible de retrouver des constructions récurrentes sur le modèle des réductions schématiques mais une majorité de ces constructions semblait constituer les parties d'une seule et même chaîne beaucoup plus longue que prévue. Le travail d'analyse montrait qu'il devait exister une forme de continuité entre au moins une vingtaine de catégories. Chose plus étonnante encore, l'analyse sémantique montrait que les catégories du modèle conceptuel tendaient à se superposer également avec les fonctions hiérarchiquement organisées dans le modèle narratif de Vladimir Propp (1970) (Figure 1).

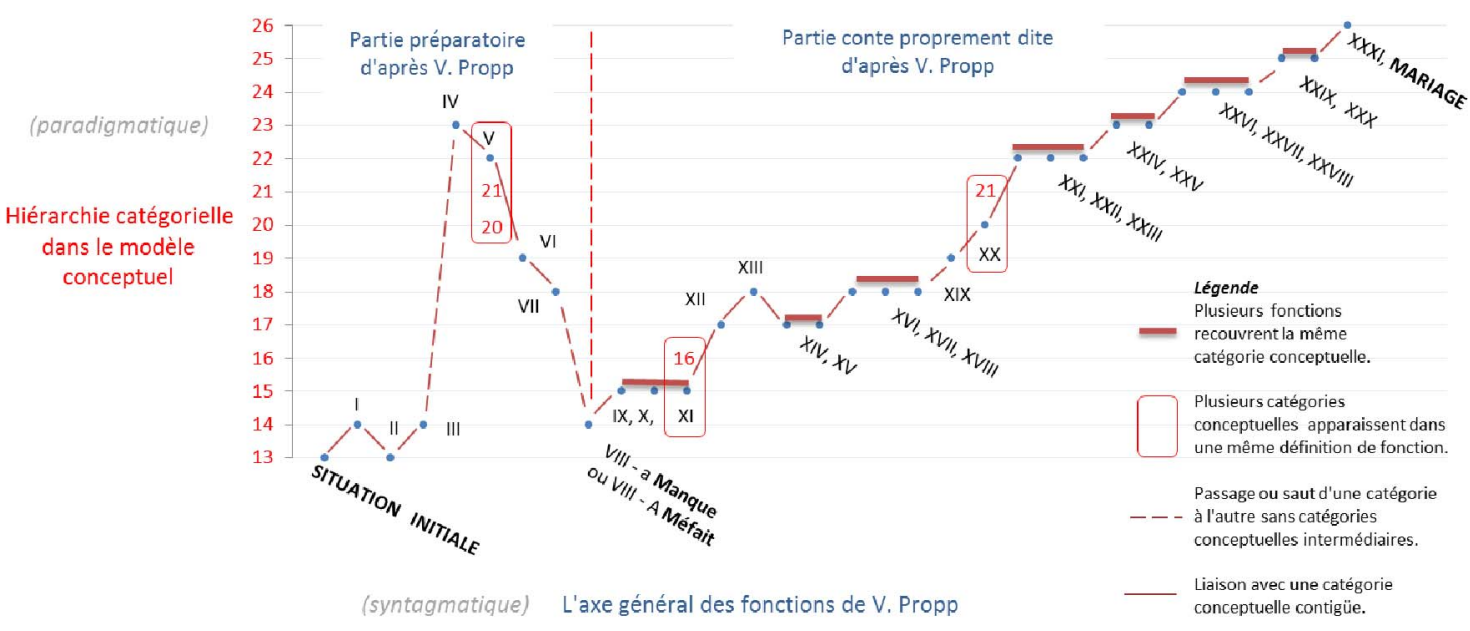

Figure 1: Correspondance entre les fonctions narratives de Vladimir. Propp et les catégories conceptuelles. A condition de regrouper certaines des fonctions de V. Propp dans le schéma narratif et d'en éclater d'autres, dans la partie conte proprement dite, et en dehors de la fonction XIII qui marque un écart par rapport à la progression générale du modèle conceptuel, les deux hiérarchies sont parallèles et isotopes.

\subsubsection{Le caractère récurrent des associations conceptuelles}

Pour visualiser l'ordonnancement conceptuel observable dans les textes, le graphe ci-dessous reproduit un des schémas étudiés. Ce graphe (Figure 2) montre une des formes que revêtent de telles séquences conceptuelles dans un texte et la façon dont elles semblent en constituer l'ossature. 


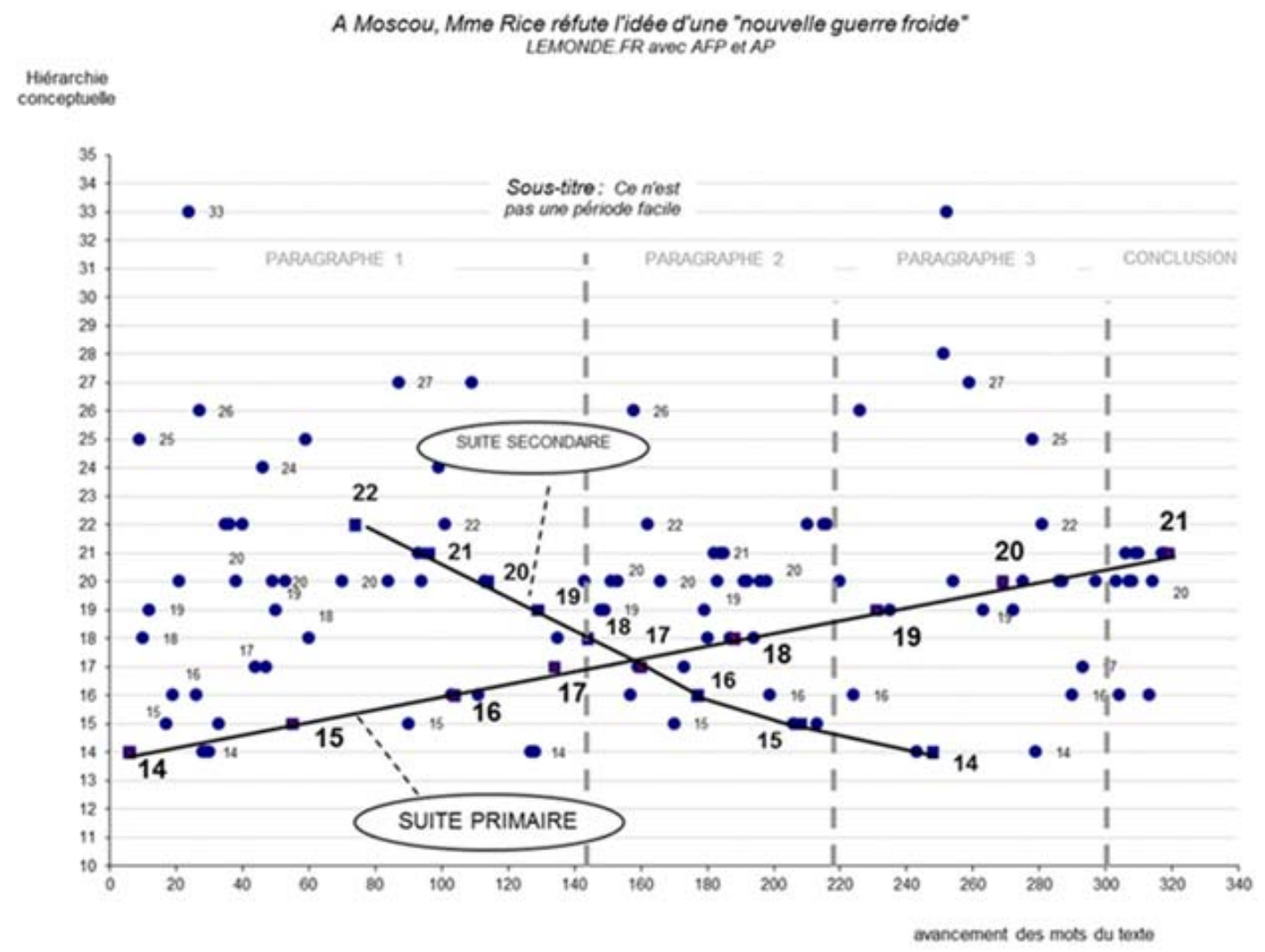

Figure 2 : Semis de points faisant apparaître des séquences métaconceptuelles régulières et ordonnées.

Sur les 322 mots que comprend cet article au total, 121 sont apparus, en première lecture, comme représentatifs d'un type dans la typologie conceptuelle. Sur la figure, chaque point correspond à une occurrence qui se rapporte de façon explicite à la définition d'un noyau de sens comme UNION, FORCE ou EVENEMENT... Sont représentés en abscisse sur le graphe la position des mots dans le texte et en ordonnées, l'indice des types correspondants. A l'analyse du graphe, on remarque la présence de deux séquences conceptuelles qui s'imbriquent, l'une croissante, l'autre décroissante. D'autres séquences plus petites ne sont pas signifiées sur le graphe. On observe enfin qu'un certain nombre d'occurrences restent isolées et ne s'inscrivent pas dans un incrément régulier.

A ce stade, une illustration semble nécessaire pour introduire les notions de concepts et d'articulation en séquences isomorphes et de la même manière mieux considérer le champ d'expressions généré par chacun de ces noyaux de sens. Cependant, avant d'aborder ce chapitre, il nous faut au préalable justifier de notre mode de traitement de la polysémie.

\subsection{Les difficultés}

Pour vérifier la présence d'un axe catégoriel dans le langage naturel, les principales difficultés provenaient de deux choses en particulier : i) de la faiblesse de la base lexicale et donc du choix du corpus pour notre thèse, et ii) du caractère polysémique des éléments du lexique. 


\subsubsection{La faiblesse de la base lexicale}

12000 lemmes référencés dans leurs catégories, flexions comprises, ne pouvaient traiter l'ensemble des styles littéraires. En effet, même s'il est généralement admis que 1000 mots suffisent pour comprendre une langue étrangère, il n'empêche que le lexique du français doit comporter quelque un million de mots et plus. Il fallait donc circonscrire notre analyse dans un premier temps à un corpus relativement simple et surtout facile d'accès en particulier numériquement. Les articles de presse en français en provenance des journaux Le Monde.fr et Courrier International.fr (type dépêche - 300 à 1200 mots) répondaient à ces conditions. Bien que les concepts furent cernés à partir de plusieurs milliers de textes et discours de toutes provenances et formes confondues (radios, télévisions, poèmes, romans, bandes dessinées, presse, textes scientifiques, religieux, etc.), en choisissant en principal un corpus journalistique pour nos expériences, nous avons conscience qu'il demeure toujours la suspicion d'analyser un corpus ad hoc, car il est généralement admis que la presse est par définition le lieu privilégié du poncif journalistique. Cependant nous relativisons cette affirmation car comme nous l'avons dit, le modèle conceptuel s'apparente aussi au modèle morphologique des contes populaires russes élaboré par Vladimir Propp.

\subsubsection{La question de la polysémie}

Mais la principale difficulté portait sur le caractère polysémique des éléments du lexique.

Il faut différentier au moins deux types de polysémie. Soit la polysémie est liée à l'occurrence rencontrée et à elle seule, c'est le cas par exemple avec le lemme transport, réductible à deux concepts types ou classes sémantiques : (i) //TRAJECTOIRE// avec le préfixe trans qui signifie 'au-delà de, à travers', et (ii) la classe //FORCE// avec le radical port qui dénote le sème /pesant/ ou /poids/; soit (et/ou) l'occurrence est interprétable en contexte comme par exemple '(faire un) tour' qui tend à se rattacher à la classe //TRAJECTOIRE// et non plus seulement à celle d'//UNION// ainsi qu'on l'a vu plus haut. Il faut dire que le modèle conceptuel présente un nombre restreint de catégories et par conséquent, d'une façon générale, chaque occurrence rencontrée dépasse rarement deux ou trois affectations catégorielles possibles. Mais à quel moment doit-on choisir l'une plutôt que l'autre ? Une façon de remédier à l'inconvénient de la polysémie était de changer d'optique et de s'appuyer sur la règle de la continuité sémique.

Changer d'optique revient à, non plus regarder la polysémie comme un handicap mais au contraire à la regarder comme une richesse. Car en réalité, peu importe qu'un objet linguistique ou tout autre objet soit multifonctionnel, ce qu'on lui demande, c'est d'abord d'être fonctionnel au moment où on en a besoin, dans la configuration imposée. Du point de vue de l'analyse, il ne s'agit plus de regarder l'objet uniquement sous l'angle de sa potentialité d'utilisation mais avant tout sous l'angle pratique de son utilisation effective. En suivant Jacques Fontanille (2004), nous pourrions dire qu'il s'agit de l'observer non comme un simulacre virtuel mais au contraire comme l'élément d'un dispositif réalisé et incarné.

Par exemple, un marteau ou une massette peuvent tout à la fois servir à enfoncer un clou comme de servir de petite enclume, tout dépendra de l'usage du moment. Il s'agit pourtant toujours du même marteau ou de la même massette mais l'utilisation dépendra de ce que l'on est en train de faire, c'est-à-dire du procès dans lequel s'inscrit l'utilisation de l'outil. En revanche, on hésitera à utiliser une perceuse électrique par exemple, comme enclume, ou comme marteau pour enfoncer un clou; à moins d'être très original. Il faut donc admettre au moins deux choses : Un objet peut être multifonctionnel et tous les objets ne répondent pas à l'utilisation demandée.

Que l'occurrence considérée contienne de la polysémie, au sens du modèle conceptuel, n'est pas incompatible avec la recherche de linéarités dans l'organisation sémique. Mais assurément, il faut pouvoir justifier de sa bonne utilisation au moment voulu. Il faudra donc qu'elle réponde à deux conditions en même temps : d'abord qu'elle contienne bien le sème recherché, et ensuite qu'elle s'insère entre deux autres unités porteuses des sèmes des catégories contigües, amont et aval. Ce faisant, nous précisons ici, nous semble-t-il, ce que Jacques Fontanille (2004: 4) considère comme «les deux tâches complémentaires de toute analyse sémiotique : 
(i) définir le niveau de pertinence optimal pour procurer à l'ensemble des observables une forme signifiante cohérente et complète, et

(ii) décrire et modéliser toutes les contraintes propres à l'objet qui est soumis à l'analyse ».

\section{Justification des récurrences metaconceptuelles}

\subsection{Le noyau de sens, un tronc commun conceptuel}

Pour illustrer notre proposition, nous prendrons trois concepts issus de notre typologie et que nous nommerons pour les besoins de la démonstration par des indices. Nous définirons dès à présent que ces trois concepts, d'indice 16, 17 et 18, s'appliquent respectivement aux idées :

a. d'union (ensemble, groupe...) type 16,

b. de force (gravité, masse, poids, pesanteur, surcroît de quelque chose...) type 17,

c. d'événement (acte posé, action, opposition, conflit, voire explosion...) type 18.

On peut d'ores et déjà noter que si ces concepts semblent a priori générer pour chacun d'eux un panel de notions relativement variées car, en effet, l'idée de surcroît de quelque chose en type 17 n'apparaît pas forcément liée à la notion de gravité, et l'idée d'explosion en type 18 non plus à celle d'action, rien n'empêche d'une part de pouvoir les regrouper de cette façon et de remarquer d'autre part, une réelle cohérence dans ces groupements d'idées d'autant que chacun des concepts évoque un champ nettement différencié des deux autres. Autrement dit, la compartimentation opérée ici semble acceptable et relativement logique.

Chacun des concepts cités en exemple étant bien différent des deux autres, la question se pose alors de savoir si l'on retrouve groupé peu ou prou ces trois types conceptuels dans des textes, dans le langage courant ou tout simplement le "bon sens ». Nous chercherons donc à montrer dans les exemples qui suivent comment ces concepts tendent généralement à s'ordonner ou, au moins, à s'associer.

\subsection{Recherche d'une récurrence générale}

Pour pouvoir qualifier ces associations de «récurrentes », nous avons étudié différents textes d'auteurs pris à des époques et dans des corpus différents. Une première recherche rapide dans l'œuvre de Saint Exupéry propose :

1) «Mes raisons pèsent, je vaincrai. C'est la pente naturelle des événements. » (Vol de Nuit)

Dans cette citation, les idées de poids ou de pesanteur (type 17) avec le mot pèse, et le mot événement (type 18) semblent éloquents et aller tout à fait dans le sens de notre proposition. De plus, il faut noter le mot vaincrai comme s'inscrivant en double dans la logique du concept 18. Car, vaincre présuppose un combat ou une opposition.

Dans la proposition 2 qui suit, Saint Exupéry associe le mot acte, au mot amour certes, mais aussi au mot querelle. Nous avons compris que ces deux occurrences, acte et querelle, relèvent du concept 18 plutôt que des deux autres concepts union(16) et force (17). Puis dans la dernière partie de la citation, il réemploie le verbe peser caractéristique du concept 17 :

2) «Je refuserai désormais de juger l'homme sur les formules qui justifient ses décisions. On se trompe trop aisément sur la caution des paroles, comme sur la direction des actes. 
Celui qui marche vers sa maison, j'ignore s'il marche vers la querelle ou vers l'amour. Je me demanderai : «Quel homme est-il ? » Alors seulement je connaîtrai vers où il pèse et où il ira. » (Antoine de Saint Exupéry - Pilote de Guerre).

La proposition 2 regroupe là encore nos deux concepts 18 et 17 . Il faut cependant noter dans cette citation l'inversion des concepts 17 et 18 .

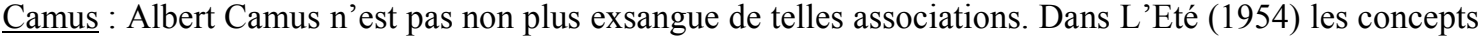
de force et le mot violence, comprenons le concept d'événement (acte posé, opposition, conflit, etc.,) sont à nouveau réunis :

3) «(...) la force et la violence sont des dieux solitaires. Ils ne donnent rien au souvenir. ».

Ailleurs, dans Lettres à un ami allemand, puis dans L'envers et l'endroit (1958), Albert Camus propose de joindre à nouveau au concept de force (type 17), les idées de lutte et d'opposition (type 18) :

4) «Qu'est-ce que l'homme ? Il est cette force qui finit toujours par balancer les tyrans et les dieux. » (Lettres à un ami allemand).

5) «Que l'espoir de vie renaisse et Dieu n'est pas de force contre les intérêts de l'homme. » (L'envers et l'endroit).

De 4, le concept de force (17) est parfaitement défini, et le concept d'événement (18) se justifie par l'occurrence finit, qui sous-tend une forme terminative, celle d'un combat finalement victorieux contre les tyrans et les dieux. De 5 enfin, le concept de force est encore parfaitement défini et le concept d'événement ressort avec la seule occurrence contre qui indique la présence d'une opposition.

A travers les exemples de ces deux auteurs français contemporains, on constate qu'il existe certes une redondance d'emploi de ce type d'association que nous cherchons à mettre en évidence mais on ne dit rien véritablement du caractère récurrent significatif, propre à confirmer notre hypothèse. Pour répondre à cette question, il nous faut poser le regard plus loin et envisager deux autres directions de recherche. La première, que nous dirons synchronique, consiste à chercher ce type d'associations conceptuelles dans des traductions récentes à partir d'autres langues. Et la seconde, diachronique, interroge l'histoire, c'est à dire quelques textes à travers les âges. Si de telles associations, dans ces conditions, se reproduisent nous pourrons conclure sans doute positivement, au moins pour ces trois concepts pris en exemple, au caractère récurrent de leur association.

\subsection{Recherche d'une récurrence en synchronie}

Comme pour la recherche diachronique, il serait vain de chercher de telles associations dans chacune des langues du Monde et dans chacun des textes édités. On peut donc restreindre, en particulier pour ce qui concerne la recherche synchronique, le domaine des investigations à une seule série de texte pris au hasard dans un même espace et une même période de temps. Le journal «Courrier International » est particulièrement indiqué pour cet exercice. Retenons uniquement la parution du premier août 2008, puis regardons là encore rapidement si certains articles de provenances diverses, associent ces mêmes types conceptuels. Après une lecture rapide nous retiendrons pour notre démonstration quatre articles significatifs parmi d'autres. Il s'agit uniquement d'articles issus de langues européennes : l'allemand, le polonais, l'espagnol et l'anglais. 
Die Welt : Dans le premier article traduit de l'allemand, Thomas Schmid du journal « Die Welt» de Berlin, écrit en page cinq de « Courrier International » :

6) «(...). Jamais l'UE ne trouvera de cohérence dans l'unité politique. Depuis que le mot malheureux de « finalité » s'est glissé dans les déclarations sur l'avenir de l'UE, la réalité européenne semble en mauvaise posture : elle ne cesse de paraître sérieusement contraire à ses idéaux. Ce faisant, nous oublions les dimensions de ce continent si longtemps divisé et qui a en outre ployé sous le fardeau et la violence des nationalismes du XIXe siècle. A tel point que la crise internationale en Belgique (...)».

Dans cet extrait, dont on remarque qu'il regroupe plusieurs phrases, les trois concepts d'indice 16, 17 et 18 sont parfaitement réunis et ordonnés. En effet, le concept d'union (ensemble, groupe...type 16) se révèle doublement, d'abord avec l'acronyme $U E$ (union européenne) deux fois présent puis, avec le mot unité. L'idée de force (pesanteur, masse, gravité, surcroît de quelque chose...type 17) s'exprime, elle, par trois occurrences significatives dans le même groupe : si longtemps, qui marque l'idée d'une période de temps excessive ; en outre, qu'il faut entendre comme, en plus; et fardeau, qui contient les sens de poids et de surcharge. Enfin, l'idée d'événement (action, opposition, conflit, voire explosion...type 18) se révèle encore une fois ici avec le mot violence (voir Camus, citation 3), ses synonymes étant : action brutale et agression. On notera dès à présent la proximité, mais dans deux phrases contigües, des mots violence et crise.

Dziennik : Le deuxième article intitulé « La vie quotidienne à la cour du président Kaczynski » en page 16 de «Courrier International » traduit du polonais et écrit conjointement par Michal Majewski et Pawel Reszka du journal «Dziennik » de Varsovie, présente dans sa conclusion la même association des trois concepts 16,17 et 18 :

7) «Le soir, il aime bavarder avec son vieil ami Maciej Lopinski. 'C'est l'un de ses plus anciens amis, ils se connaissent de l'époque où ils étaient des opposants', précise un politicien important de Droit et justice. 'Ils sirotent du vin et évoquent le bon vieux temps'. »

Dans cet article, bien que seulement suggérée par la préposition avec, on ne peut exclure la présence du concept 16 d'union car le texte indique clairement que les deux amis se retrouvent "ensemble » le soir. Le concept 17 de force est évoqué par le superlatif, l'un de ses plus, qui exprime ici l'idée d'une «force » d'amitié particulière. Enfin, le concept 18 révèle sa présence parfaite avec l'occurrence opposants. Comme pour l'article de Die Welt, on notera que ces trois concepts apparaissent à nouveau non seulement dans le même ordre que la typologie conceptuelle proposée mais également de façon indépendante des ruptures phrastiques. Il faut remarquer enfin que l'opération de débrayage qui institue un discours de second degré, ne semblent pas non plus affecter l'ordre conceptuel.

El Païs : Mauricio Vincent dans «El Païs » de Madrid au sujet d'un article sur Cuba (page 17 de Courrier International) nous propose à nouveau dans deux extraits de son article, d'ordonner d'une part les concepts 16,17 et 18 , puis 17 et 18 :

8) «La célébration était lourde de symbole : le nouveau président cubain [...] s'est exprimé devant une image géante de Fidel Castro et il n'a eu de cesse de répéter que les temps s'annonçaient difficiles du fait de la crise mondiale (...) ». 
Dans cet extrait le concept d'union (groupe, ensemble, etc.), révèle indirectement sa présence à partir de l'occurrence célébration; indirectement car on est obligé d'imaginer en effet, dans la scène proposée, la foule des spectateurs et des journalistes venue écouter le nouveau président cubain. Le concept de force est présent grâce à deux occurrences principales, le mot lourdes et l'itération n'a eu de cesse de répéter, en référence respectivement aux idées d'insistance ou d'un surcroît d'effort nécessaire. L'idée d'événement ou d'acte posé enfin, en concept 18, s'exprime en principal ici avec le mot fait (du latin factum, la chose faite). Il est appuyé, sur chacun de ses côtés par les mots difficiles qui indique généralement la présence d'une épreuve ou d'une opposition, et crise - déjà repéré dans la citation de Die Welt (6) - car son emploi, bien qu'il relève dans ce cas précis d'un contexte économique comme dans l'extrait précédent d'ailleurs, peut cependant aussi aller jusqu'à évoquer le conflit ou le combat notamment sur le plan médical, lorsqu'il s'agit d'une lutte entre un agent d'agression et les forces de défense de l'organisme.

Le deuxième extrait beaucoup plus court, dans le même article, évoque d'abord l'idée d'un surcroît de quelque chose ou une augmentation, idée typique du concept 17, avec l'expression a ajouté, puis d'opposition voire de conflit (18) avec le mot choc:

\section{9) (...). Raul Castro a ajouté que pour amortir le choc, il était (...).}

Le mot choc peut porter à s'interroger mais, faut-il préciser qu'il signifie une rencontre violente et aussi un combat. D'ailleurs, à propos de la bataille de Normandie, le général de Gaulle ne disait-il pas en s'adressant aux français sur radio Londres : "Après tant de souffrances (...), voici venu le choc décisif ». Remarquons là encore la même construction conceptuelle, l'idée d'un surcroît de quelque chose avec, tant de souffrances et l'idée d'un combat avec le mot choc.

The Economist : Enfin pour clore cette série d'exemples, en page 26 de « Courrier International », « The Economist » de Londres propose un article dont l'introduction recèle une magnifique suite des trois concepts 16,17 et 18 :

10) «Le premier septembre 2008 se tiendra à Bruxelles le premier sommet européen consacré aux Roms. Cette initiative, qui réunira les représentants des institutions européennes, des Etats et de la société, se penchera sur les discriminations de plus en plus aiguës dont font l'objet les Tsiganes - notamment en Italie, où les procédés utilisés par les autorités rappellent les heures les plus sombres de l'histoire européenne ».

Comment ne pas reconnaitre, maintenant qu'on a un peu plus l'habitude, les trois concepts encore une fois parfaitement réunis et ordonnés ; le type 16 avec le mot réunira appuyé par la présence de deux groupes parfaitement signifiés, les Roms d'un côté et les représentants de l'autre; le type 17 avec de plus en plus; et le type 18 avec le syntagme les procédés utilisés qui dénote, sans ambiguité, son caractère factuel.

Il est bien évident que ces cas ne sont pas isolés et que nous n'avons choisi de n'en présenter que quatre ou cinq parmi d'autres dans ce numéro de "Courrier International », ceux-là précisément parce que plusieurs de ces exemples, comme de ceux qui vont suivre dans la recherche sur la récurrence en diachronie, révèlent plus que ces trois concepts. Pour le moment il s'agit d'en saisir les principes en considérant l'amplitude et la variété des champs d'expression qu'ils sous-tendent.

\subsection{Recherche d'une récurrence en diachronie}

La recherche de la récurrence en synchronie nous a montré que les associations conceptuelles présentaient un caractère non systématique mais redondant dans des textes contemporains écrits ou traduits en français. Dans la recherche de la récurrence diachronique nous allons chercher à remonter le temps pour 
savoir si ce type d'association s'observe également à travers les siècles. Pour cette démonstration, peu d'exemples suffisent. Nous en choisirons six seulement, deux au XIXe siècle, un au XVIIIème siècle, un autre au XVIIème et enfin, après un bond de près de deux mille ans dans l'histoire, deux dans l'Antiquité.

\subsubsection{Du romantisme à la période classique,}

Victor Hugo : Ainsi, en 1831, dans Notre Dame de Paris, livre X chapitre IV, l'auteur écrit :

11) «Cependant les gueux ne se décourageaient pas. Déjà plus de vingt fois l'épaisse porte sur laquelle ils s'acharnaient avait tremblée sous leur bélier de chêne multipliée par la force de cent hommes. Les panneaux craquaient les ciselures volaient en éclats ( ...)».

Puis plus loin

12) «Tout à coup, au moment où ils se groupaient pour un dernier effort autour du bélier, chacun retenant son haleine et raidissant ses muscles afin de donner toute sa force au coup décisif, un hurlement, plus épouvantable que celui qui avait éclaté et expiré sous le madrier, s'éleva au milieu d'eux ».

Dans 11 et 12, une fois encore l'ordre conceptuel est respecté. Dans la première citation, on ne peut pas exclure le concept 16 puisque «les gueux» forment un groupe. Ensuite l'idée de force (17) relève de trois occurrences consécutives: plus de vingt fois (itératif), l'épaisse porte (pesanteur, puissance) et force. Enfin, craquaient et les ciselures volaient en éclats marquent le caractère terminatif de l'action, spécifique du concept 18 .

Dans la citation 12, Victor Hugo nous amène au même processus et ajoute en plus, explicitement l'occurrence groupaient, typique du concept 16. Le concept 17 se présente quant à lui sous la forme d'une double occurrence, effort puis force; enfin, le concept 18, relève de trois occurrences à caractère terminatif, coup décisif, éclaté et expiré. On notera en outre la similitude d'emploi de l'expression coup décisif avec choc décisif dans l'explication de la citation 9.

Montesquieu : On note ce type de construction également chez Vigny, Balzac, Zola et tant d'autres auteurs encore du XIXe siècle. De la même manière, on le retrouvera au XVIIIe Siècle. Montesquieu par exemple, dans son livre XI Chapitre VI de L’Esprit des lois, précise :

13) « Il serait inutile que le corps législatif fût toujours assemblé. Cela serait incommode pour les représentants, et d'ailleurs occuperait trop la puissance exécutrice, qui ne penserait point à exécuter, mais à défendre ses prérogatives, et le droit qu'elle a à exécuter ».

Comment ici encore ne pas reconnaître les trois idées que nous cherchons à mettre en évidence, l'idée de groupe ou d'ensemble avec le mot assemblée, l'idée de force avec le mot puissance et l'idée d'opposition, de poser un acte, avec les mots exécuter et défendre. On ne peut que constater, qu'il s'agisse du XVIIIe Siècle, du XIXe ou d'aujourd'hui, cette construction est pour le moins toujours redondante.

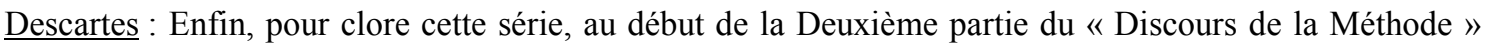
(Principales règles de la méthode), Descartes regroupe et ordonne à nouveau nos trois concepts. Dans la citation ci-dessous, le concept 16 (union, ensemble groupe) s'appuie en principal sur le syntagme avec mes pensées, le concept 17 relève des occurrences aux aspects fréquentatif et quantitatif, respectivement, 
souvent et pas tant, et le concept 18 se retrouve une nouvelle fois révélée par l'occurrence fait (voir citation 8). Mais, comme on peut le remarquer dans cet extrait de Descartes, des propositions entières suggèrent également l'existence d'une construction conceptuelle globale et réflexive, autrement dit, intellectuellement ordonnée.

14) Descartes en 1637 propose l'énoncé suivant :

a. «J'étais en Allemagne, (...), je demeurais tout le jour enfermé seul dans un poêle, où j'avais tout le loisir de m'entretenir avec mes pensées.

b. Entre lesquelles l'une des premières fut que je m'avisais de considérer que souvent il n'y a pas tant de perfection

c. dans les ouvrages composés de plusieurs pièces, et faits de la main de divers maîtres, qu'en ceux auxquels un seul a travaillé ».

Bien que beaucoup d'autres exemples chez Descartes fussent encore plus simples d'analyse, il semblait utile et intéressant de prendre celui-ci pour mieux montrer les différents niveaux d'analyse possibles.

\subsubsection{La période Antique}

Faisons maintenant un bond en arrière. $\mathrm{Si}$, de la période Contemporaine à la période Classique on retrouve ce même type de construction alors, permettons-nous un bond dans l'histoire de près de deux mille ans. Sautons la période de la Renaissance et aussi celle du Moyen-âge pour laquelle on sait déjà qu'au IXème siècle l'héritage grec n'était pas complètement perdu mais était sans doute mal connu et mal compris en Europe. Beaucoup de textes anciens en effet ne furent redécouverts qu'à partir du début du Xème siècle. La pensée philosophique et scientifique du monde chrétien dès lors allait pouvoir bénéficier de toute une littérature traduite de l'arabe, autrement dit d'un double héritage : celui des travaux originaux des Arabes eux-mêmes et des nombreux textes grecs que ces derniers avaient su préserver. Dans ces conditions il faut enfoncer des portes ouvertes en rappelant que notre pensée moderne est empreinte des deux courants à la fois, grecque et arabe et sans aucun doute également, nombre de nos constructions intellectuelles. Aussi pour clore cette démonstration, allons directement rechercher chez Plutarque d'abord, cet écrivain grec qui vécut au premier siècle (vers 45 - vers 125) puis, chez Platon, plus ancien encore $(427-348 / 347$ av. J.C.) une dernière trace de ces associations conceptuelles.

Plutarque : Dans ses « Euvres morales » Plutarque, s'interrogeant sur les moyens de réprimer la colère, propose une très belle suite d'abord entrelacée pour les concepts 18 et 17 mais dont la structure générale est au final parfaitement inversée :

15) «(...) c'est là un acte de force puissante et victorieuse, de force luttant contre la passion non pas avec des nerfs et des muscles, mais avec l'énergie de la volonté. C'est pourquoi je m'étudie constamment à recueillir et à étudier non seulement les exemples laissés par ces philosophes que les gens sensés disent n'avoir point de fiel, mais encore, et plus volontiers, ceux des monarques et des tyrans ». (traduction de Ricard)

C'est là un acte dit-il (concept 18), de force puissante (deux fois le concept 17), de force (17), luttant contre (deux fois 18) puis, il termine un peu plus loin par le concept 16 avec l'occurrence principale recueillir, sorte de glomérule, qu'il appuie en nommant à la suite cinq occurrences de types groupe (16) : les exemples, les philosophes, les gens sensés, les monarques et les tyrans. 


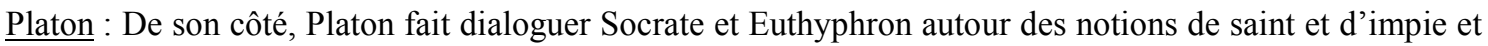
Socrate de proposer à Euthyphron :

16) «Et si nous contestions sur la pesanteur, notre différend ne serait-il pas bientôt terminé par le moyen d'une balance ». (Traduction de Victor Cousin, 1822)

Platon, ou plutôt son traducteur, propose ici une suite de 3 concepts consécutifs depuis le nous qui marque la présence d'un groupe (type 16) jusqu'au mot terminé qui marque virtuellement l'accomplissement de l'acte (type 18). Mais, ce qui est remarquable de constater, c'est que le texte associe le concept d'opposition (type 18) donné par différend et terminé au mot précis de pesanteur (type 17). Bref, ces deux concepts 17 et 18 sont associés de façon tellement impromptue qu'il est légitime a priori d'éliminer la thèse d'une complicité consciente de l'auteur pour grouper ces concepts. De telles associations « impromptues » sont très fréquentes. De fait, elles ne peuvent pas toujours être dues au hasard, d'autant que précisément ici, dans cette citation de Platon, on retrouve une occurrence typique du concept 19 avec le mot balance, occurrence que nous avons déjà rencontrée dans la citation 4 chez Camus :

(Citation 4): «Qu'est-ce que l'homme ? Il est cette force qui finit toujours par balancer les tyrans et les dieux. » (Lettres à un ami allemand).

\section{L'analyse}

Alors, que peut-on conclure à propos du caractère récurrent de ces associations conceptuelles ? Manifestement à la vue de ces exemples, il semble difficile d'exclure cette possibilité, que ce soit dans le langage d'aujourd'hui ou dans les citations d'hier. Il semble en effet qu'après vingt-quatre siècles, nous retrouvions encore et toujours les mêmes groupements conceptuels, au moins pour ce que nous avons vu, c'est à dire les trois types conceptuels considérés ici, Union, Force, Evénement. Nous aurions pu tout aussi bien interroger d'autres textes ou les textes sacrés où, là également ces associations sont courantes. Mais l'illustration semblait suffisante.

On peut toujours objecter maintenant que ces citations ont été choisies ad hoc. Cependant toutes furent analysées comme du tout-venant, soit à partir d'un seul et même journal, soit au cours d'une recherche expresse sur quelques publications ou sur l'Internet. Nous comprenons toutefois qu'il est légitime de s'interroger d'une part, sur le mode de catégorisation mais nous l'avons défini au départ, et de ce point de vue il n'est pas à remettre en cause ${ }^{7}$ même s'il peut arriver parfois que certains items puissent recouvrir plusieurs concepts en même temps, et d'autre part, de s'interroger sur le caractère accidentel de ces associations car elles ne sont pas systématiques, nous l'avons dit. En d'autres termes ces associations relèvent-elles d'un caractère fortuit, c'est-à-dire sont-elles dues purement au hasard de l'agencement des mots ou bien existe-t-il une logique ou une loi qui tend malgré tout à les assembler ? Existe-t-il une loi telle, que le caractère récurrent, que nous avons tenté d'illustrer, nous fasse sortir du résultat ordinaire des probabilités ? Certes, dans certains cas, on ne peut pas nier l'existence du hasard. D'ailleurs des études statistiques montrent clairement qu'on ne peut pas l'exclure totalement mais, en revanche, ces mêmes études montrent aussi qu'il existe toute une part non équivoque qui sort de la moyenne statistique.

Récapitulons. A partir des trois concepts d'indice 16, 17 et 18, nous avons d'abord cherché à montrer qu'on pouvait ramener à un tronc commun de ressemblance conceptuelle des expressions très différentes, par exemple qu'une situation de type 16 (concept d'Union) était repérables soit par le mot ensemble, soit par un acronyme comme UE (Union Européenne - citation 6), soit encore par l'idée de groupe «au moment où ils se groupaient » (Victor Hugo, citation 12); autrement dit, qu'il existait des concepts repérables à partir de formes les plus diverses (verbes, substantifs, adjectifs, acronymes, syntagmes, etc.), ce qui a amené à considérer l'étendue du panel d'occurrences possibles permettant de repérer ces concepts dans les textes. Nous avons également remarqué, qu'un concept ne se révélait pas uniquement 
par des occurrences toujours précises mais parfois aussi par des images ou des suggestions d'auteurs. Ainsi, lorsque Mauricio Vincent dans «El Païs » de Madrid, emploie le mot célébration (citation 8), il suggère indirectement l'existence d'un groupe (type 16) mais, dans ce cas, le sème /groupe/ en effet, n'est qu'afférent, selon la terminologie de F. Rastier (1987: 133).

Au-delà des primitives qui leur sont affectées pour les nommer (Union, Force, Evènement), nous avons vu également que ces concepts présentaient des caractéristiques aspectuelles (voire casuelles); par exemple, les unités discursives qui se rapportent au concept d'Union, lequel sous-tend les idées d'ensemble et de groupe, possèdent fréquemment la marque du pluriel ; celles du concept de Force se révèlent préférablement par certains aspects itératif ou fréquentatif; enfin, celles du concept d'Evénement se révèlent plutôt à partir de traits de type factuel ou terminatif.

Nous venons d'énumérer certaines caractéristiques d'aspect, bien que les items considérés le fussent indépendamment du verbe. Le concept de Force par exemple relève dans certains cas d'une forme itérative " n'a eu de cesse de répéter " (citation 8), et le concept d'Evénement relève plutôt d'une forme d'accomplissement. On peut bien sûr ramener, par comparaison, la sémantique des metaconcepts à un trait grammatical associé au prédicat c'est-à-dire indiquant la façon dont le procès ou l'état exprimé par le verbe est envisagé du point de vue de son développement (commencement, déroulement, achèvement, évolution globale ou au contraire moment précis de cette évolution, etc.), mais on voit bien que les métaconcepts s'articulent indépendamment des phrases et, par conséquent, qu'ils ne devraient pas être soumis au procès du verbe dans la phrase. En d'autres termes, ils devraient être indépendants ou plus exactement antérieurs au sujet de l'énonciation.

Enfin, nous avons pu constater qu'il n'apparaissait pas, en général, de sélection objective entre deux occurrences consécutives relevant d'une même séquence, c'est-à-dire déterminante au sens de L. Hjelmslev (1996). Et pourtant la syntaxe conceptuelle était parfaitement respectée. C'est le cas de nombre d'articles dans notre illustration; notamment dans l'article de «Courrier International » traduit du polonais :

(Citation 7) «Le soir, il aime bavarder avec son vieil ami Maciej Lopinski. 'C'est l'un de ses plus anciens amis, ils se connaissent de l'époque où ils étaient des opposants', précise un politicien important de Droit et justice. 'Ils sirotent du vin et évoquent le bon vieux temps'. »

Dans cet extrait les trois concepts sont présents et ordonnés malgré l'absence a priori de causalité, Le soir, il aime bavarder avec son vieil ami, concept d'ensemble (16), C'est l'un de ses plus, concept 17 de force ou de surcroît de quelque chose, et enfin, défiant encore toute logique de cause à effet, l'auteur utilise le mot opposants, occurrence générique en concept 18. Rien, dans cet extrait, ne montre qu'effectivement l'auteur suit une progression logique, donc a priori causale et pourtant, il emploie bien l'ordre typologique du Modèle Conceptuel.

Nous croyons que c'est l'ensemble de ces observations qui permet de conclure à l'existence d'une cause récurrente derrière le raisonnement conscient; une certaine «logique», ou «loi», qui somme toutes s'apparenterait à certains algorithmes de l'intelligence artificielle tant les chemins qu'elle emprunte semblent repérables et modélisables.

\section{Conclusion}

Le caractère hiérarchique du modèle conceptuel pose, on le voit, beaucoup de questions et, comme on peut s'en douter également, il en pose aussi dans des domaines très différents, syntaxe, sémantique, lexicologie, neurosciences, etc... Mais, en définitive..., en pose-t-il davantage qu'il ne permettrait peutêtre d'en résoudre? Car l'existence d'une contrainte hiérarchique de catégories sémantiques «fondamentales» semble permettre de considérer sous un éclairage nouveau plusieurs des théories linguistiques européennes et anglo-saxonnes. A condition de ne chercher à observer dans un premier 
temps qu'un niveau très profond qui, sortant des limites de la phrase, se différentie radicalement du formalisme grammatical (sujet, verbe, objet...) ou du nœud verbal et donc encore de la recherche de connexions entre proto-actants positionnels selon Jean Petitot (1985: 140 et suiv.), le modèle conceptuel semble moins porter une théorie nouvelle qu'à chaque fois un complément de réponse aux différentes théories structurales existantes.

Ce n'est pas que le modèle conceptuel rejette sujet ou agent. Non, car il existe une réalité du sujet, pensé formellement et anthropomorphisé ou pensant et agissant, qui appartient au domaine du sensible, c'est le fameux cogito. Mais, le locuteur apparaît, avec le modèle conceptuel, comme contraint dans son discours par une sorte de surdétermination qui le dépasse donc, l'oblige. C'est dans ce sens que le modèle conceptuel interroge la syntaxe et la sémantique fondamentale ${ }^{8}$. Et si l'on considère maintenant que seuls, sujet et prédicat, participent de la pleine conscience du locuteur alors, et puisque les catégories sémantiques s'articulent indépendamment des césures phrastiques, nous pouvons aussi sans doute poser, au moins à titre d'hypothèse, l'existence d'un niveau sémantico-syntaxique antérieur (protosyntaxique et protosémantique) au pouvoir génératif, et indépendant du raisonnement conscient.

De toute évidence, s'il existe bien une hiérarchie catégorielle contraignante et transphrastique qui s'exonère au moins dans un premier temps du sujet et de l'objet primo-actants, alors, devrait s'ouvrir un espace linguistique, sémiotique et cognitif nécessairement riche de nombreuses perspectives.

\section{Références bibliographiques}

Chomsky N. (1971). Aspects de la théorie syntaxique, Paris, Le Seuil.

Dehaene St. (2007). Les Neurones de la lecture. Paris : éd. Odile Jacob, 182-187.

Desclés JP. (1994). Réflexions sur les grammaires cognitives.http://alic.parissorbonne.fr/PUBLICATIONS/descles/modeles94.pdf, 9.

Fontanille J. (2004), Pratiques Sémiotiques Immanence et Pertinence des Textes aux Pratiques, http://www.unilim.fr/pages_perso/jacques.fontanille/textes-pdf/CPratiques_semiotiques2004_06.pdf, 4.

Greimas A.J. \& Courtès J. (1987). Dictionnaire raisonné de la théorie du langage, Paris, Hachette, 33.

Hjelmslev L. (1996). Prolégomènes à une théorie du langage, Paris, De Minuit, 39 et 57.

Petitot J. (1991). Syntaxe topologique et grammaire cognitive. L'objet sens et réalité. In: Langages, $n^{\circ}$ 103, 97-128

Petitot J. (1985). Morphogenèse du sens, Paris, PUF.

Petitot J. (1991). Syntaxe topologique et grammaire cognitive. L'objet sens et réalité. In: Langages, $n^{\circ}$ 103, 97-128

Petitot J. (1992). Physique du sens, Paris, CNRS.

Pottier B. (1992). Sémantique générale, Paris, PUF (Ch. VII: Concepts, noèmes et universaux).

Propp V. (1970). Morphologie du conte, Paris, Le Seuil, 35-81.

Rastier F. (1987). Sémantique Interprétative, Paris, PUF.

Victorri, B. (1994). La construction dynamique du sens. http://halshs.archives-ouvertes.fr/halshs-00139120, 1.

Wierzbicka A. (1996). Primes and Universals, Oxford, University Press.

\footnotetext{
${ }^{1}$ Pour Jean Petitot (1991), il existe une convergence entre le point de vue morphodynamique sur les structures sémiolinguistiques et certaines orientations maîtresses des sciences cognitives. Il précise en particulier "que le tournant cognitif de la linguistique conduit à des problèmes nouveaux, totalement inédits, ayant plus à voir avec des
} 
problèmes de physique, d'analyse du signal ou d' 'information processing' qu'avec des problèmes traditionnels de linguistique (...)».

${ }^{2}$ Ibidem page 121 ; à propos des travaux de René Thom sur la syntaxe topologique.

${ }^{3}$ Peut-être d'essence biologiques : une partie de la thèse est consacrée à montrer les analogies entre ce que Stanislas Dehaene (2007) nomme recyclage neuronal et propriétés non-accidentelles, et les catégories fondamentales du modèle conceptuel.

${ }^{4}$ Dans ce sens, nous nous associons à Jean-Pierre Desclés (1994: 9) lorsqu'il critique chez R. Langacker sa « logique formelle ». Dans ces approches logico-linguistiques, dit-il, " aucune relation n'est vraiment posée entre un niveau conceptuel et figuratif, d'une part et un niveau d'expressions linguistiques, d'autre part; le langage y est conçu comme une activité autonome plus ou moins coupée de toute autre activité cognitive (...)».

${ }^{5}$ Par exemple des lexies comme passer, aller, chemin, processus, à travers, en haut, en bas, etc. dénotent le même sème /trajectoire/, lequel s'oppose aux sèmes /union/ et /force/.

${ }^{6}$ Par rapport au modèle conceptuel, nous entendons le mot incarné dans un sens fonctionnel, c'est-à-dire dans une interaction dynamique entre tout et parties.

${ }^{7}$ F. Rastier (1987: 19) rappelle qu'il existe des qualités définitoires et comme telles ne peuvent être remises en cause. On peut seulement chercher à les élucider.

8 Pour A.J. Greimas et J. Courtès (1979: 380), «La syntaxe fondamentale constitue, avec la sémantique fondamentale, le niveau profond de la grammaire sémiotique et narrative. Elle est censée rendre compte de la production, du fonctionnement et de la saisie des organisations syntagmatiques, appelées discours, (...): elle représente donc l'instance ab quo du parcours génératif de ces discours ». 